International Journal of Applied Mathematics

Volume 34 No. $2 \quad 2021,377-389$

ISSN: 1311-1728 (printed version); ISSN: 1314-8060 (on-line version)

doi: http://dx.doi.org/10.12732/ijam.v34i2.13

\title{
BOUNDARY VALUE PROBLEM FOR LOADED EQUATION OF PARABOLIC-HYPERBOLIC TYPE OF THE THIRD ORDER IN AN INFINITE THREE-DIMENSIONAL DOMAIN
}

\author{
Bozor I. Islomov ${ }^{1}$, Yolqin K. Alikulov ${ }^{2}$ \\ ${ }^{1}$ National University of Uzbekistan \\ named after Mirzo Ulugbek \\ Prosp. Universitet, 4 \\ Tashkent - 100125, UZBEKISTAN \\ 2 Tashkent University of Information Technologies \\ named after M. Al-Khworazmi \\ Prop. Amir Temur, 108 \\ Tashkent - 100200, UZBEKISTAN
}

\begin{abstract}
In this paper, it is formulated and studied one of the problems for the loaded of parabolic-hyperbolic type equations of the third order in an infinite three-dimensional domain. The main method for study of the formulated problem is the Fourier transform. The uniqueness and existence of a regular solution of the problem are proved.
\end{abstract}

AMS Subject Classification: 35M10, 35M12, 35L10, 35K10, 42B10 Key Words: third-order equation; loaded equation; Fourier transform; regular solution; extremum principle; solution estimate

\section{Introduction}

One of the important classes of non-classical equations of mathematical physics is an equation of composite and mixed-composite type, involved elliptic, elliptichyperbolic and parabolic-hyperbolic operators. Correct formulated boundary

Received: December 20, 2020

(C) 2021 Academic Publications

${ }^{\S}$ Correspondence author 
value problems for elliptic-hyperbolic and parabolic-hyperbolic type equations of the third order were first studied in the works of A.B. Bitsadze and M.S. Salakhitdinov [1], M.S. Salakhitdinov [2], T.D. Dzhuraev [3]. In these works, in order to study boundary value problems, the representation of the general solution of an equation of mixed-composite type in the form of a sum of functions was used. Such a representation plays an important role for equations formed from the product of permutational differential operators. Further, there are various types of the third-order partial differential equations developed by [4], [5] in this area. Many important problems of mathematical physics and biology [6], especially the problems of long-term forecasting and regulation of groundwater, the problem of heat and mass transfer with a finite velocity, the movement of a low-compressible fluid surrounded by a porous medium, optimal control of an agroecosystem, lead to boundary value problems for loaded partial differential equations. In 1969 A.M. Nakhushev proposed a number of problems of new types, which are closely related to loaded differential equations [7] called boundary value problems with the displacement in mathematical literatures.

Definition 1. A differential equation $A u(x)=f(x)$, given in a $n$ dimensional domain $\Omega$ of Euclidean space of points $x=\left(x_{1}, x_{2}, \ldots, x_{n}\right)$, is called loaded, if it contains a trace of some operations from the desired solution $u(x)$ on the closure $\bar{\Omega}$ of manifolds of dimension less than $n$.

Definition 2. The equation is called a loaded differential equation in a domain $\Omega \subset R^{n}$, if it contains at least one derivative of the required solution $u(x)$ on manifolds of nonzero measure belonging to $\bar{\Omega}$.

Main loaded differential and integro-differential equations in a domain $\Omega \in$ $R^{n}$ can be represented as in [8]

$$
\mathcal{A} u(x) \equiv \mathcal{L} u(x)+\mathcal{M} u(x)=f(x),
$$

where $\mathcal{L}$ is differential operator, and $\mathcal{M}$ is differential, integral or integrodifferential operator, respectively, including the operation of taking a trace from the desired solution $u(x)$ on manifolds of nonzero measure belonging to $\bar{\Omega}$ (measure is strictly less than $n$ ). For example, loaded problems of vibrations with lumped masses are reduced to the simplest equation of the form of a sum of operators which are widely applied in physics and technology in [9]. Note that the presence of a loaded operator $M$ requires researcher of additional considerations on the application of the well-known theory of boundary value problems for differential equations of the form $\mathcal{L} u(x)=f(x), x \in \Omega$. On the other 
hand, the problem of finding solutions of loaded differential equations in predetermined classes can lead to new problems for unloaded differential equations (1). Local and nonlocal problems for second-order partial differential equations of hyperbolic and mixed types with loaded terms in special domains were considered in [10], [11]. Boundary value problems for the loaded hyperbolic, mixed and mixed-composite types of the third order partial differential equations are still poorly investigated. This is due, first of all, to the absence of representation of the general solution for such differential equations. On the other hand, such problems are reduced to few studied integral and integro-differential equations with a shift (see, in [12]). The theory of boundary value problems for loaded second order equations with an integro-differential operator was studied in [13], [14].

After the publication of the well-known works by A.V. Bitsadze [15] many mathematicians began to study boundary value problems for mixed elliptichyperbolic type equations in three-dimensional domains. A number of research papers appeared (see, for example [16], [17]), where three-dimensional analogues of the Tricomi, Gellerstedt, and Keldysh problems for second order equations of mixed type were considered. Boundary value problems for a second-order mixed parabolic-hyperbolic equation in a three-dimensional prismatic domain were studied in [18]. We note, that the boundary value problems for the loaded third-order differential equations of hyperbolic, mixed and mixed-composite types in infinite three-dimensional domain were few studied (in [19], [20]).

In this paper, we study local problem for the loaded parabolic-hyperbolic type equation of the third order in an infinite three-dimensional domain.

\section{Problem Formulation}

Consider the equation

$$
\mu U(x, 0, z)=\frac{\partial}{\partial y}\left[\frac{1-\text { signy }}{2} U_{y y}+\frac{1+\text { signy }}{2} U_{y}-U_{x x}-U_{z z}\right],
$$

where

$$
\mu=\text { const }<0 .
$$

The three dimensional domain $\Omega$ is bounded by the following surfaces: $\Gamma_{0}$ : $x=0, \quad 0 \leq y \leq h, \quad z \in R, \quad \Gamma_{1}: x=1, \quad 0 \leq y \leq h$,

$z \in R, \quad \Gamma_{2}: y=h, \quad 0 \leq x \leq 1, \quad z \in R, \quad S_{1}: x+y=0, y \leq 0$, $0 \leq x \leq \frac{1}{2}, \quad z \in R, \quad S_{2}: x-y=1, \quad y \leq 0, \quad \frac{1}{2} \leq x \leq 1, \quad z \in R$.

Let us introduce the notation 


$$
\begin{gathered}
\Omega_{1}=\Omega \cap\{(x, y, z): x>0, \quad y>0, \quad z \in R\}, \\
\Omega_{2}=\Omega \cap\{(x, y, z): x>0, \quad y<0, \quad z \in R\}, \\
I=\{(x, y, z): 0<x<1, y=0, \quad z \in R\}, D=\Omega \cap\{z=0\}, \\
D_{j}=\Omega_{j} \cap\{z=0\}, \sigma_{j}=S_{j} \cap\{z=0\} \quad(j=1,2), \\
\gamma_{l}=\Gamma_{l} \cap\{z=0\}(l=\overline{0,2}), J=I \cap \quad\{z=0\} .
\end{gathered}
$$

Problem $A$. Find the function $U(x, y, z)$ with the following properties:

1) $U(x, y, z)$ is continuous up to the boundary of the domain $\Omega$;

2) $U(x, y, z) \in C^{1}\left(\Omega \cup S_{1} \cup S_{2}\right)$;

3) $U_{x x y}(x, y, z), U_{z z y}(x, y, z) \in C\left(\Omega_{1} \cup \Omega_{2}\right), U_{y y}(x, y, z) \in C\left(\Omega_{1}\right)$, $U_{y y y}(x, y, z) \in C\left(\Omega_{2}\right)$ and the equation (2) is satisfied in the domains $\Omega_{j}(j=$ $1,2)$;

4) $U(x, y, z)$ satisfies the conditions

$$
\begin{gathered}
\left.U\right|_{\Gamma_{0}}=\Psi_{0}(y, z),\left.\quad U\right|_{\Gamma_{1}}=\Psi_{1}(y, z), \quad 0 \leq y \leq h, \\
\left.U\right|_{S_{1}}=\Psi_{2}(x, z),\left.\quad \frac{\partial U}{\partial n}\right|_{S_{1}}=\Psi_{3}(x, z), \quad 0 \leq x \leq \frac{1}{2}, \quad z \in R, \\
\left.\frac{\partial U}{\partial n}\right|_{S_{2}}=\Psi_{4}(x, z), \quad \frac{1}{2} \leq x \leq 1, \quad z \in R, \\
\lim _{|z| \rightarrow \infty} U=\lim _{|z| \rightarrow \infty} U_{x}=\lim _{|z| \rightarrow \infty} U_{y}=\lim _{|z| \rightarrow \infty} U_{z}=0,
\end{gathered}
$$

where $\Psi_{j}(\bullet, z)-$ are given sufficiently smooth functions such that

$$
\Psi_{0}(0, z)=\Psi_{2}(0, z), \lim _{|z| \rightarrow \infty} \Psi_{j}(\bullet, z)=0 \quad(j=\overline{0,4}) .
$$

\section{Investigation of Problem $A$}

According to the condition of Problem $A$, its solutions can be represented in the form of the Fourier integral, namely,

$$
U(x, y, z)=\frac{1}{\sqrt{2 \pi}} \int_{-\infty}^{+\infty} u(x, y, \lambda) e^{-i \lambda z} d \lambda .
$$


Applying the Fourier transform (8) to the equation (2) we obtain following equation

$$
\frac{\partial}{\partial y}\left[\frac{1-\text { signy }}{2} u_{y y}+\frac{1+\text { signy }}{2} u_{y}-u_{x x}+\lambda^{2} u\right]=\mu u(x, 0, \lambda),
$$

where $\lambda \in R$.

By virtue of (8), Problem $A$ is equivalently reduced to the following plane problem.

Problem $A_{\lambda}$. Find a function $u(x, y, \lambda)$ such that:

1) $u(x, y, \lambda) \in C(\bar{D}) \cap C^{1}\left(D \cup \sigma_{1} \cup \sigma_{2}\right)$;

2) $u_{y}(x, y, \lambda), u_{x x y}(x, y, \lambda) \in C\left(D_{1} \cup D_{2}\right), u_{y y}(x, y, \lambda) \in C\left(D_{1}\right)$,

$u_{y y y}(x, y, \lambda) \in C\left(D_{2}\right)$ and the equation (9) is satisfied in the domains $D_{j}(j=$ 1,2);

3) $u(x, y, \lambda)$ satisfies the conditions:

$$
\begin{gathered}
\left.u\right|_{\gamma_{0}}=\psi_{0}(y, \lambda),\left.u\right|_{\gamma_{1}}=\psi_{1}(y, \lambda), \quad 0 \leq y \leq h, \\
\left.u\right|_{\sigma_{1}}=\psi_{2}(x, \lambda),\left.\quad \frac{\partial u}{\partial n}\right|_{\sigma_{1}}=\psi_{3}(x, \lambda), \quad 0 \leq x \leq \frac{1}{2}, \quad \lambda \in R, \\
\left.\frac{\partial u}{\partial n}\right|_{\sigma_{2}}=\psi_{4}(x, \lambda), \quad \frac{1}{2} \leq x \leq 1, \quad \lambda \in R
\end{gathered}
$$

where $\psi_{j}(\bullet, \lambda)(j=\overline{0,4})$ are given functions such that

$$
\begin{gathered}
\psi_{0}(0, \lambda)=\psi_{2}(0, \lambda), \quad \psi_{j}(\bullet, \lambda)=\frac{1}{\sqrt{2 \pi}} \int_{-\infty}^{+\infty} \Psi_{j}(\bullet, z) e^{i \lambda z} d z, \\
\psi_{i}(y, \lambda) \in C[0 ; h] \cap \cap C^{1}(0, h)(i=0,1), \\
\psi_{2}(x, \lambda) \in C^{1}\left[0, \frac{1}{2}\right] \cap C^{3}\left(0, \frac{1}{2}\right), \\
\psi_{3}(x, \lambda) \in C\left[0, \frac{1}{2}\right] \cap C^{2}\left(0, \frac{1}{2}\right), \\
\psi_{4}(x, \lambda) \in C\left[\frac{1}{2}, 1\right] \cap C^{2}\left(\frac{1}{2}, 1\right) .
\end{gathered}
$$

Any regular solutions of equation (9) are represented as (see, in [1]):

$$
u(x, y, \lambda)=v(x, y, \lambda)+\omega(x, \lambda),
$$

where $v(x, y, \lambda)$ is a solution of the equation

$$
\frac{1-\operatorname{signy}}{2} v_{y y}+\frac{1+\text { signy }}{2} v_{y}-v_{x x}+\lambda^{2} v=\mu v(x, 0, \lambda), \quad \lambda \in R
$$

and $\omega(x, \lambda)=\omega_{0}(x, \lambda)$ at $0 \leq x \leq 1, y>0, \omega(x, \lambda)=\omega_{1}(x, \lambda)$ at $0 \leq x \leq \frac{1}{2}, y<0, \omega(x, \lambda)=\omega_{2}(x, \lambda)$ at $\frac{1}{2} \leq x \leq 1, y<0$, here $\omega_{l}(x, \lambda)(l=\overline{0,2})$ 
are arbitrary twice continuously differentiable functions and without loss of generality we can assume that

$$
\omega_{l}(1, \lambda)=0(l=0,2), \omega_{j}(0, \lambda)=\omega_{j}\left(\frac{1}{2}, \lambda\right)=0(j=0,1) .
$$

By virtue of representation (14), Problem $A_{\lambda}$ is reduced to Problem $A_{\lambda}^{*}$ of finding regular solution $v(x, y, \lambda)$ to equation (15) in the domain $D$ and satisfies following conditions:

$$
\begin{gathered}
\left.v\right|_{\gamma_{0}}=\psi_{0}(y, \lambda),\left.\quad v\right|_{\gamma_{1}}=\psi_{1}(y, \lambda), \quad 0 \leq y \leq h, \quad \lambda \in R, \\
\left.v\right|_{\sigma_{1}}=\psi_{2}(x, \lambda)-\omega_{1}(x, \lambda), \quad 0 \leq x \leq \frac{1}{2}, \quad \lambda \in R, \\
\left.\frac{\partial v}{\partial n}\right|_{\sigma_{1}}=\psi_{3}(x, \lambda)+\frac{1}{\sqrt{2}} \omega_{1}^{\prime}(x, \lambda), \quad 0 \leq x \leq \frac{1}{2}, \quad \lambda \in R, \\
\left.\frac{\partial v}{\partial n}\right|_{\sigma_{2}}=\psi_{4}(x, \lambda)-\frac{1}{\sqrt{2}} \omega_{2}^{\prime}(x, \lambda), \quad \frac{1}{2} \leq x \leq 1, \quad \lambda \in R,
\end{gathered}
$$

where $\omega_{i}(x, \lambda)(i=\overline{0,2})$ are unknown functions.

Applying the method used in [12], any regular solution of equation (15) can be represented as

$$
v(x, y, \lambda)=w(x, y, \lambda)+\vartheta(x, \lambda),
$$

where $w(x, y, \lambda)$ is a solution of the following equation

$$
0=\left\{\begin{array}{l}
L_{1} w \equiv w_{y}-w_{x x}+\lambda^{2} w, \quad(x, y, \lambda) \in D_{1}, \\
L_{2} w \equiv w_{y y}-w_{x x}+\lambda^{2} w, \quad(x, y, \lambda) \in D_{2},
\end{array}\right.
$$

and $\vartheta(x, \lambda)$ is a solution of the following ordinary second order differential equation

$$
\vartheta^{\prime \prime}(x, \lambda)-\left(\lambda^{2}-\mu\right) \vartheta(x, \lambda)=-\mu w(x, 0, \lambda), \quad 0<x<1
$$

Remark 3. Taking into account that the function $a_{j} \operatorname{ch} \lambda x+b_{j} \operatorname{sh} \lambda x(j=$ $\overline{0,2}$ ) satisfies equation (22), we assume that, without loss of generality,

$$
\vartheta(0, \lambda)=\vartheta(1, \lambda)=0
$$

is satisfied, in investigation of Problem $A_{\lambda}^{*}$. 
The solutions of problems (23) and (24) can be represented as

$$
\begin{aligned}
& \vartheta(x, \lambda)=\frac{\mu \operatorname{sh}(x-1) \sqrt{\lambda^{2}-\mu}}{\sqrt{\lambda^{2}-\mu} \operatorname{sh} \sqrt{\lambda^{2}-\mu}} \int_{0}^{1} \operatorname{sh} t \sqrt{\lambda^{2}-\mu} w(t, 0, \lambda) d t \\
& -\frac{\mu}{\sqrt{\lambda^{2}-\mu}} \int_{x}^{1} \operatorname{sh}(x-t) \sqrt{\lambda^{2}-\mu} w(t, 0, \lambda) d t, \quad 0 \leq x \leq 1 .
\end{aligned}
$$

By virtue of representation (21) and taking (24) into account, Problem $A_{\lambda}^{*}$ is reduced to Problem $\tilde{A}_{\lambda}^{*}$ for finding a regular solution, where $w(x, y, \lambda)$ is the solution of the equation (22) in the domain $D$. This solution should satisfy the following conditions:

$$
\begin{aligned}
& \left.w\right|_{\gamma_{0}}=\psi_{0}(y, \lambda),\left.\quad w\right|_{\gamma_{1}}=\psi_{1}(y, \lambda), \quad 0 \leq y \leq h, \quad \lambda \in R, \\
& \left.w\right|_{\sigma_{1}}=\psi_{2}(x, \lambda)-\omega_{1}(x, \lambda)-\vartheta(x, \lambda), \quad 0 \leq x \leq \frac{1}{2}, \quad \lambda \in R, \\
& \left.\frac{\partial w}{\partial n}\right|_{\sigma_{1}}=\psi_{3}(x, \lambda)-\left(\omega_{1}^{\prime}(x, \lambda)+\vartheta^{\prime}(x, \lambda)\right) / \sqrt{2}, \quad 0 \leq x \leq \frac{1}{2}, \\
& \left.\frac{\partial w}{\partial n}\right|_{\sigma_{2}}=\psi_{4}(x, \lambda)+\left(\omega_{2}^{\prime}(x, \lambda)+\vartheta^{\prime}(x, \lambda)\right) / \sqrt{2}, \quad \frac{1}{2} \leq x \leq 1,
\end{aligned}
$$

where $\vartheta(x, \lambda)$ is determined from $(25)$.

In order to prove the uniqueness of the solution for the problems $A_{\lambda}, A_{\lambda}^{*}$ and $\tilde{A}_{\lambda}^{*}$ the following theorem plays an important role.

Theorem 4. If $\psi_{0}(y, \lambda) \equiv \psi_{1}(y, \lambda) \equiv 0, \forall y \in[0, h]$, $\psi_{2}(x, \lambda) \equiv \psi_{3}(x, \lambda) \equiv 0, \forall x \in\left[0, \frac{1}{2}\right]$ and $\psi_{4}(x, \lambda) \equiv 0, \forall x \in\left[\frac{1}{2}, 1\right], \lambda \in R$, then $w(x, 0, \lambda)=\tau(x, \lambda) \equiv 0, \quad \forall x \in \bar{J}, \lambda \in R$.

Proof. Let the function $w(x, y, \lambda)$ be the twice continuously differentiable solution of the homogeneous problem $\tilde{A}_{\lambda}^{*}$ in the domains $D_{2}$ and $D_{2}^{\varepsilon}$, where $D_{2}^{\varepsilon}$ is domain with bound $\partial D_{2}^{\varepsilon}=\bar{J}^{\varepsilon} \cup \bar{\sigma}_{1}^{\varepsilon} \cup \bar{\sigma}_{2}^{\varepsilon}$ strictly lying in the domain $D_{2}$. Here, $\varepsilon$ is a sufficiently small positive number.

Integrating with the domain $D_{2}^{\varepsilon}$, the following identity

$$
0=w L_{2} w=\frac{\partial}{\partial x}\left(w w_{x}\right)-\frac{\partial}{\partial y}\left(w w_{y}\right)-\lambda^{2} w^{2}-w_{x}^{2}+w_{y}^{2}
$$

is satisfied, and applying Green's formula, we have 


$$
\int_{\bar{\sigma}_{1}^{\varepsilon} \cup \bar{\sigma}_{2}^{\varepsilon} \cup \bar{J} \varepsilon} w w_{x} d y+w w_{y} d x=\iint_{D_{2}^{\varepsilon}}\left(w_{x}^{2}-w_{y}^{2}+\lambda^{2} w^{2}\right) d x d y .
$$

Passing to limit as $\varepsilon \rightarrow 0$ in [3], we obtain

$$
\begin{gathered}
\int_{0}^{1} \tau(x, \lambda) \nu(x, \lambda) d x \\
=-w^{2}(C, \lambda)-\iint_{D_{2}}\left(w_{x}^{2}-w_{y}^{2}+\lambda^{2} w^{2}\right) d x d y,
\end{gathered}
$$

where $w(C, \lambda)=w\left(\frac{1}{2},-\frac{1}{2}, \lambda\right)=-\omega_{1}\left(\frac{1}{2}, \lambda\right)-\vartheta\left(\frac{1}{2}, \lambda\right)$,

$$
\tau(x, \lambda)=w(x, 0, \lambda), x \in \bar{J}, \nu(x, \lambda)=w_{y}(x, 0, \lambda), x \in J .
$$

We used the fact that

$$
w(0,0, \lambda)=w(1,0, \lambda)=0 .
$$

when the formula (32) is obtained.

In order to calculate the first integral on the right-hand side of equality (32), we pass to the characteristic coordinates $\xi=x-y, \eta=x+y$. Moreover, the domain $D_{2}$ goes into a triangle $\Delta^{1}$ with the sides $A C, C B$ and $B A$ which lies down on straight lines $\eta=0, \xi=\frac{1}{2}$ and $\eta=\xi$, respectively. Considering canonical form of the equation (22): $v_{\xi \eta}=\frac{\lambda^{2}}{4} v(24)$ and taking $(27)$ for $\psi_{2}(x, \lambda) \equiv 0$ into account, from the first integral on the right-hand side of equality (32) taking (16), (24), (34) and (27) into account for $\psi_{2}(x, \lambda) \equiv 0, \forall x \in\left[0, \frac{1}{2}\right]$, we have

$$
\iint_{D_{2}}\left(w_{x}^{2}-w_{y}^{2}\right) d x d y=-w^{2}(C, \lambda)-\lambda^{2} \iint_{D_{2}} w^{2} d x d y .
$$

By virtue of (35), from (32) we obtain

$$
\int_{0}^{1} \tau(x, \lambda) \nu(x, \lambda) d x=0 .
$$

According to the conditions of Problem $\tilde{A}_{\lambda}^{*}$, passing to the limit in the equation $w_{y}-w_{x x}+\lambda^{2} w=0$ as $y \rightarrow+0$, taking (33) into account, we have

$$
\nu(x, \lambda)=\tau^{\prime \prime 2} \tau(x, \lambda), \quad(x, 0) \in J, \quad \lambda \in R .
$$

Substituting (37) into (36), taking into account $\tau(0, \lambda)=\tau(1, \lambda)=0$, we obtain 


$$
\int_{0}^{1} \tau(x, \lambda) \nu(x, \lambda) d x=-\int_{0}^{1} \tau^{\prime 2}(x, \lambda) d x-\lambda^{2} \int_{0}^{1} \tau^{2}(x, \lambda) d x \leq 0 .
$$

Comparing (36) and (38), we have $\int_{0}^{1} \tau(x, \lambda) \nu(x, \lambda) d x=0$, or

$$
\int_{0}^{1} \tau^{\prime 2}(x, \lambda) d x+\lambda^{2} \int_{0}^{1} \tau^{2}(x, \lambda) d x=0
$$

Hence, it follows that $\tau(x, \lambda) \equiv 0, x \in \bar{J}, \lambda \in R$. Theorem 4 is proved.

By virtue of $(30)$ taking $\tau(x, \lambda)=w(x, 0, \lambda),, x \in \bar{J}$, into account from (25), we obtain

$$
\vartheta(x, \lambda) \equiv 0, \quad \forall x \in \bar{J}, \quad \lambda \in R
$$

Theorem 5. If the conditions of Theorem 1 and (3),(39) are satisfied, then the solution of Problem $A$ for equation (2) is unique in the domain $\Omega$.

Proof. To prove the uniqueness of the solution of Problem $A$, we first prove the uniqueness of the solution of Problems $A_{\lambda}, A_{\lambda}^{*}$ and $\tilde{A}_{\lambda}^{*}$ for equations (9), (15) and (22), respectively.

According to the maximum principle of parabolic equations (see, in [18] and $[21])$, the first boundary value problem for equation (22) in the domain $D_{1}$ with homogeneous conditions (26) and (30) (see Theorem 1) have no nonzero solution, those $w(x, y, \lambda) \equiv 0$ in $\bar{D}_{1}$. Then it follows from conditions (27)-(29) in view of (39) that

$$
\omega_{j}(x, \lambda) \equiv 0, \quad(x, 0) \in \bar{J}_{j}(j=\overline{0,2}), \quad \lambda \in R .
$$

Due to the uniqueness of solution of the Cauchy-Goursat problem with homogeneous conditions $(27)$ and $w(x, 0, \lambda)=0,(x, 0) \in \bar{J}$ for equation $(22)$ and considering (39) and (40), we obtain $w(x, y, \lambda) \equiv 0$ in $\bar{D}_{2}$. Therefore,

$$
w(x, y, \lambda) \equiv 0, \quad(x, y) \in \bar{D}, \quad \lambda \in R
$$

Hence, the solution of Problem $\tilde{A}_{\lambda}^{*}$ for equation (22) is unique.

Taking (39), (41) and the conditions of Theorem 1 into account from (21), we obtain 


$$
v(x, y, \lambda) \equiv 0, \quad(x, y) \in \bar{D}, \quad \lambda \in R .
$$

This proves the uniqueness of the solution to Problem $A_{\lambda}^{*}$ for equation (15). Further, by virtue of (40) and (42), from (14) we have

$$
u(x, y, \lambda) \equiv 0, \quad(x, y) \in \bar{D}, \quad \lambda \in R .
$$

The uniqueness of the solution of Problem $A_{\lambda}$ for equation (9) follows from (43). We suppose that $\Psi_{0}(y, z) \equiv \Psi_{1}(y, z) \equiv \Psi_{2}(x, z) \equiv \Psi_{3}(x, z) \equiv \Psi_{4}(x, z) \equiv$ 0 and (7) holds. Then, by virtue of (6) and (43) from (8) follows that

$$
U(x, y, z) \equiv 0,(x, y, z) \in \bar{\Omega} .
$$

Thus, the uniqueness of the solution of Problem $A$ for equation (2) is proved, i.e. Theorem 5 is proved.

Theorem 6. If the conditions $\omega_{1}(x, \lambda), \omega_{2}(x, \lambda), \vartheta(x, \lambda), \psi_{1}(y, \lambda), \psi_{3}(x, \lambda), \psi_{4}(x$, $O\left(\frac{1}{|\lambda|^{k}}\right), k>3, \vartheta^{\prime}(y, \lambda), \omega_{1}^{\prime}(y, \lambda)$, $\psi_{0}(y, \lambda), \quad \psi_{2}^{\prime}(x, \lambda)=O\left(\frac{1}{|\lambda|^{k} c h|\lambda|}\right), \quad \psi_{2}(x, \lambda)=O\left(\frac{c h|\lambda|}{|\lambda|^{k+1} \cdot t h|\lambda|}\right)$, hold, then, the function $u(x, y, \lambda)$ satisfies the following estimate for large values of parameter $|\lambda|$ :

$$
u(x, y, \lambda)=O\left(1 /|\lambda|^{k}\right), \quad k>3 .
$$

Using the extremum principle in [18] for parabolic and hyperbolic equations and the method used in [16], Theorem 6 is proved.

The existence of the solution of Problem $A$ is proved by the method of integral equations. In order to prove the existence of the solution of Problem $A$, we first prove the existence of the solution of Problem $A_{\lambda}$ for equation (9).

Theorem 7. If conditions (3), (12) and (13) are satisfied, then the solution of Problem $A_{\lambda}$ for equation (9) in the domain $D$ exists.

Theorem 7 is proved by the method given in [11], [19].

Theorem 8. Let the solution $u(x, y, \lambda)$ of the plane Problem $A_{\lambda}$ for equation (9) exist and be given by the formula

$$
u(x, y ; \lambda)=\frac{1}{\sqrt{2 \pi}} \int_{-\infty}^{+\infty} U(x, y, z) e^{i \lambda z} d z
$$


and for large values of $|\lambda|$ estimate (45) hold. Then in the domain $\Omega$ the solution of Problem $A$ for equation (2) exists and it is found by formula (8), and vice versa.

Remark 9. The estimate (45) is proved for the existence of integral (8) giving a solution of Problem $A$.

Remark 10. Using the properties of the Fourier transform from [22], we can prove the validity of estimates (6), that is, $U(x, y, z), U_{x}(x, y, z), U_{y}(x, y, z)$, $U_{z}(x, y, z)$ tend to zero when $|z| \rightarrow \infty$ and all of the corresponding improper integrals exist.

Remark 11. Theorem 5 implies the equivalence of Problems $A$ and $A_{\lambda}$.

\section{References}

[1] A.V. Bitsadze, M.S. Salakhitdinov, On the theory of equations of mixedcomposite type, Siberian Math. J., II, No 1 (1961), 7-19.

[2] M.S. Salakhitdinov, Mixed-Compound Equations, Fan, Tashkent (1974).

[3] T.D. Dzhuraev, Boundary Value Problems for Equations of Mixed and Mixed-Composite Type, Fan, Tashkent (1979).

[4] S.X. Chen, Mixed type equations in gas dynamics, Quart. Appl. Math., LVXIII, No 3 (2010), 487-511.

[5] B.I. Islomov, B.Z. Usmanov, Nonlocal boundary value problem for a thirdorder equation of elliptic-hyperbolic type, Lobachevskii J. of Math., 41, No 1 (2020), 32-38.

[6] A.M. Nakhushev, Equations of Mathematical Biology, Higher School, Moscow (1995).

[7] A.M. Nakhushev, Bias Problems for Partial Differential Equations, Science, Moscow (2006).

[8] M.T. Dzhenaliev, M.I. Ramazanov, Loaded Equations as Perturbations of Differential Equations, FAST, Almaty (2010). 
[9] A.M. Nakhushev, Loaded Equations and Their Applications, Nauka, Moscow (2012).

[10] K.B. Sabitov, Initial boundary value problem for a parabolic-hyperbolic equation with loaded terms, Izvestiya Vuzov Math., 6 (2015), 31-42.

[11] B.I. Islomov, U.I. Baltaeva, Boundary value problems for a third-order loaded parabolic-hyperbolic equation with variable coefficients, EJDE, 2015 (2015), 1-10.

[12] B.I. Islomov, D.M. Kuryazov, Boundary value problems for a mixed loaded third-order equation of parabolic-hyperbolic type, Uzbek. Mathematical J., 2 (2000), 29-35.

[13] B.S. Kishin, O.Kh. Abdullaev, About a problem for loaded parabolichyperbolic type equation with fractional derivatives, Internat. J. of Diff. Equations, 6 (2016), 1-7.

[14] O.Kh. Abdullaev, B.I. Islomov, Gellerstedt type problem for the loaded parabolic-hyperbolic type equation with Caputo and Erdélyi-Kober operators of fractional order, Izvestiya Vuzov Math., 64, No 10 (2020), 29-42.

[15] A.V. Bitsadze, On equations of mixed type in three-dimensional domains, Reports of the USSR Acad. of Sci., 143 (1962), 1017-1919.

[16] A.M. Ezhov, S.P. Pulkin, Estimation of the solution of the Tricomi problem for one class of equations of mixed type, Reports of the USSR Acad. of Sci., 193, No 5 (1970), 978-980.

[17] M.S. Salakhitdinov, B.I. Islomov, Boundary value problems for an equation of mixed type with two internal degeneration lines, Reports of the USSR Acad. of Sci., 316, No 5 (1991), 1051-1054.

[18] T.D. Dzhuraev, A. Sopuev, M. Mamazhonov, Boundary Value Problems for Equations of Parabolic-Hyperbolic Type, FAN, Tashkent (1986).

[19] T.K. Yuldashev, B.I. Islomov, E.K. Alikulov, Boundary-value problems or loaded third-order parabolic-hyperbolic equations in infinite threedimensional domains, Lobachevskii J. of Math., 41, No 5 (2020), 922-940.

[20] B.I. Islomov, G.B. Umarova, Three-dimensional problems for a parabolichyperbolic equation with two planes of change of type, Lobachevskii J. of Math., 41, No 9 (2020), 1811-1822. 
[21] A.M. Ilyin, A.S. Kalashnikov, O.A. Oleinik, Linear equations of the second order of parabolic type, Zh. Vychisl. Math. and Math. Phys., 4, No 6 (1965), 1006-1024.

[22] S.G. Samko, A.A. Kilbas, O.I. Marichev, Integrals and Derivatives of Fractional Order and Some of Their Applications, Nauka, Tekhnika, Minsk (1987); 2 Ed. in EN: Fractional Integrals and Derivatives: Theory and Applications, Gordon and Breach, Yverdon (1993). 
